\title{
Sodium Bensuldazate
}

National Cancer Institute

\section{Source}

National Cancer Institute. Sodium Bensuldazate. NCI Thesaurus. Code C75208.

The sodium salt form of bensuldazic acid, a veterinary antifung al agent. 